\title{
Using Short Messages to Encourage COVID-19 Prevention Behaviors
}

\author{
Sophia L. Pink, ${ }^{1 *}$ Michael N. Stagnaro, ${ }^{1,2}$ James Chu, ${ }^{1}$ Joseph Mernyk, ${ }^{1}$ Jan G. Voelkel, ${ }^{1}$ \\ and Robb Willer ${ }^{1}$ \\ ${ }^{1}$ Department of Sociology, Stanford University \\ ${ }^{2}$ Sloan School of Management, Massachusetts Institute of Technology
}

Date: April 13, 2021

Note: This is a working paper that has not been peer reviewEd.

*Corresponding author: Sophia L. Pink (sopink@stanford.edu). 


\begin{abstract}
Controlling the spread of COVID-19 requires persuading the mass public to change their behavior in significant ways. Many efforts to encourage behavior change, such as public service announcements, social media posts, and billboards, involve short, persuasive appeals, yet the effectiveness of these messages is unknown. Here, we test whether short messages increase intentions to comply with public health guidelines. Research was conducted in the United States from March-July 2020. To identify promising messages, we conducted two pretests (total $\mathrm{N}=$ 1,596) where participants rated the persuasiveness of 56 unique messages: 31 based on the persuasion and social influence literature and 25 from a pool of 600 crowdsourced messages by online respondents. The four top-rated messages emphasized 1) civic responsibility to reciprocate the sacrifices of health care workers, 2) caring for the elderly and vulnerable, 3) a specific, sympathetic victim, and 4) limited health care system capacity. We then conducted three well-powered, pre-registered experiments (total $\mathrm{N}=3,719$ ) testing whether these four toprated messages and a standard public health message based on language from the CDC increased intentions to comply with public health guidelines. In Study 1, we find the four messages and the standard public health message significantly outperformed a null control. In Studies 2 and 3, we compared the effects of persuasive messages to the standard public health message, finding that none consistently out-performed the standard public health message. Short messages can increase intentions to comply with public health guidelines, but short messages featuring persuasive techniques from the social science literature did not substantially outperform standard public health messages.
\end{abstract}

Keywords: COVID-19, persuasion, communication, public health, messaging 


\section{Using Short Messages to Encourage COVID-19 Prevention Behaviors}

The coronavirus pandemic has infected more than 93 million people globally, claimed the lives of more than 2 million people, severely affected economic growth and employment in nearly every country, and changed everyday life as we know it. Despite the availability of viable vaccines, bottlenecks in distribution and production and the proliferation of new variants of the virus, mean that widespread behavioural compliance to public health guidelines remains critically important (1). Moreover, the reopening of restaurants, stores, and other businesses is contingent on overall levels of engagement in preventative behaviours. To ensure engagement in preventative behaviours, such as wearing a face covering and physical distancing, organizations and governments will need to ensure that public health messages are compelling and persuasive.

A substantial body of work preceding the pandemic has tested whether short messages -such as those found on social media, public service announcements, or billboards -- can impact people's health behaviours $(2,3,4)$. Likewise, researchers have studied how to create messages that persuade people to engage in behaviours to protect their own and others' health $(5,6)$ and to reduce risk (7). Further, beyond public health, there is a vast, multidisciplinary literature on effective persuasive techniques (8).

Nevertheless, it is unclear the extent to which short messages are persuasive in the context of the COVID-19 pandemic in the United States, and if so, which persuasive strategies are most effective at promoting behaviour change. The COVID-19 pandemic differs in many ways from recent public health crises -- it directly impacts almost every American's life, requires sudden and drastic behaviour change, and is occurring in a deeply polarized country with historically-low trust in media (9) and government (10). People have been saturated with often conflicting messaging from a variety of news sources, the federal government, local and state 
governments, employers, schools, friends and family. Consistent with this, views of the coronavirus pandemic have polarized along political lines (11), reported skepticism about the seriousness of COVID-19 is high, and conspiracy theories regarding its origins have proliferated (12).

To test the efficacy of short messages, we first conducted preliminary tests of 56 short messages collected from a review of past literature and online crowdsourcing. To identify messages with the highest potential impact, we asked respondents to rate the perceived persuasiveness of each message. Second, we tested the effects of the four top-rated persuasive messages from the pre-test, as well as a standard message based on messaging used by the Centers for Disease Control, on intentions to engage in preventive behaviours relative to no messages. Finally, we conducted a pair of experiments to directly compare the effectiveness of the four top-rated persuasive messages with the standard public health message.

\section{Short Messages as a Medium of Persuasion}

Short messages are one of the most important forms of persuasion available for agencies seeking to increase public health compliance. There are many media through which persuasive appeals occur, but short messages, such as short radio scripts, pre-roll ads, text messages, or text on billboards, are ubiquitous. Research shows that short messages, which are scalable and consumable even to those with short attention spans, are key in reaching the general public (13). A meta-analysis of mass-media public health campaigns involving short messages found that messaging campaigns lead to approximately a $5 \%$ increase in the number of people performing the intended behaviour (2). Other reviews have also found that mass media campaigns have small to moderate effects on health behaviours (14), and that short messages can impact 
behaviours related to physical activity, sexual health, treatment-seeking, and smoking cessation $(3,4)$.

However, the context of the COVID-19 pandemic differs from the contexts of much of this past research. First, it requires sudden, difficult behaviour change by the majority of people in the United States, such as avoiding visiting close family members. Second, there is widespread skepticism about the dangers of the virus and the effectiveness of preventative behaviours, such as mask-wearing. Third, people are receiving constant information about COVID-19 from many sources, so short messages may have a small or negligible effect. Given this unique context, it is important to test whether any short messages can impact prevention behaviours.

\section{Persuasive Strategies}

Many studies have been conducted across the behavioural and social sciences investigating which persuasive strategies used in short messages are most effective at encouraging public health behaviours. In addition to having practical implications, short messages allow strong theoretical tests, because it is easier to control language in a short message than other formats, such as longer videos or in-person conversations. This literature spans many disciplines, making it difficult to review comprehensively. However, there are several messaging approaches and debates that are common to much of the literature about prevention behaviors.

For example, past public health research has found that prosocial messages are more likely to change people's behaviour than self-interesting messaging $(15,16)$. However, other work has shown that self-interested messages are just as effective, if not more effective, in contexts such as vaccinations (17). Past research has also found that stories of identifiable 
victims are more persuasive that statistics about larger groups (18). In addition, social proof -indicating that many other people are complying with guidelines -- has been shown to be effective for prompting behavior change (19). Similarly, reciprocity messaging -- the desire to help people who have helped you -- is another approach that has been shown to be effective for persuasion (20). An additional approach is moral messaging -- appealing to an individual's moral foundations (e.g. harm/care, authority) can convince people to change their mind on polarizing issues, such as climate change (21) universal healthcare, military spending, and other politicallycharged topics (22). Researchers have also begun to analyze which type of persuasive messaging are most effective within the context of the COVID-19 pandemic $(16,23,24)$. However, we do not know the relative impact of all of these messages, because the studies have been conducted using different outcome behaviors, different contexts, and different sample populations.

\section{The Present Research}

The studies that follow have three main objectives. Our first objective was to systematically identify messages with high potential for persuasiveness from a large set of possible messages. In two pre-tests (total $\mathrm{N}=1,596$ ), we tested how persuasive participants found 31 messages based on public health research and 25 messages drawn from 600 crowdsourced messages, for a total of 56 messages.

Our second objective was to test whether reading a single persuasive message would impact intentions to engage in COVID-19 preventative behaviours, compared to a null control (i.e., seeing no message). In Study 1, which was pre-registered, we examined whether the four top-rated messages from the pre-tests and a standard public health message increased intentions to engage in preventive behaviours. We tested the messages in a between-subjects format, using 
the difference between people's reported past behaviours before seeing the message and intended future behaviours after seeing a message as the dependent variable.

Our third objective was to test whether specific persuasive strategies can outperform a standard public health message with a description of the virus and prevention behaviours. Using a standard message as an "active control", we can isolate the effects of a particular messaging approach, independent of the effect of receiving a reminder of the virus and desirable responses. In Studies 2 and 3, which were pre-registered, we test whether any of the top-performing persuasive messages from the pre-tests could out-perform a standard public health message.

\section{Pretests}

Our goal for the pretests was to identify promising messages to test further.

\section{Method}

In the first pretest of 598 participants, we measured perceptions of the persuasiveness of 24 short messages aimed at convincing people to stay home to prevent the spread of COVID-19. In the second pre-test of 998 participants, we used this same method, but added 7 additional messages from literare and 25 messages crowdsourced from participants in the first pre-test. (sSe Supplementary Materials for details).

The messages were based on a wide range of both general and prior research on persuasion and messaging, and research specific to the current pandemic. All messages were two to four sentences long and advocated for staying home to prevent the spread of coronavirus. See Supplementary Materials for message text. The control message stated, "Coronavirus is a respiratory illness that can spread from person to person. The virus is thought to spread mainly between people who are in close contact with one another. You can help prevent the spread of 
COVID-19. Stay home and avoid contact with others when you must go out." Each participant rated 10 messages.

\section{Results}

We used a mixed-effects model to calculate main effects of message condition on persuasiveness, with a control message as the reference. The level 1 effect was the message, and the level 2 effect was the participant. We included controls for gender, age, race, education, and income. The outcome variable was the beta value associated with each message, nonstandardized. We used the Holm method to adjust p-values to account for multiple comparisons. In a merged analysis of the two pre-tests, the five most persuasive messages emphasized responsibility to reciprocate sacrifices of healthcare workers (Message $\# 6, \beta=0.54, p_{\text {holm }}<$ .001 ), risk of overwhelming healthcare resources (Message $\# 15, \beta=0.46, p_{\text {holm }}<.001$ ), the story of a sympathetic victim (Message $\# 7, \beta=.32, p_{\text {holm }}<.001$ ), and protecting the vulnerable (Message $\# 0, \beta=.29, p_{\text {holm }}<.001$ ), and the speed of transmission (Message $\# 8, \beta=0.29$, $\left.p_{\text {holm }}<.001\right) .($ See Table 1.) 


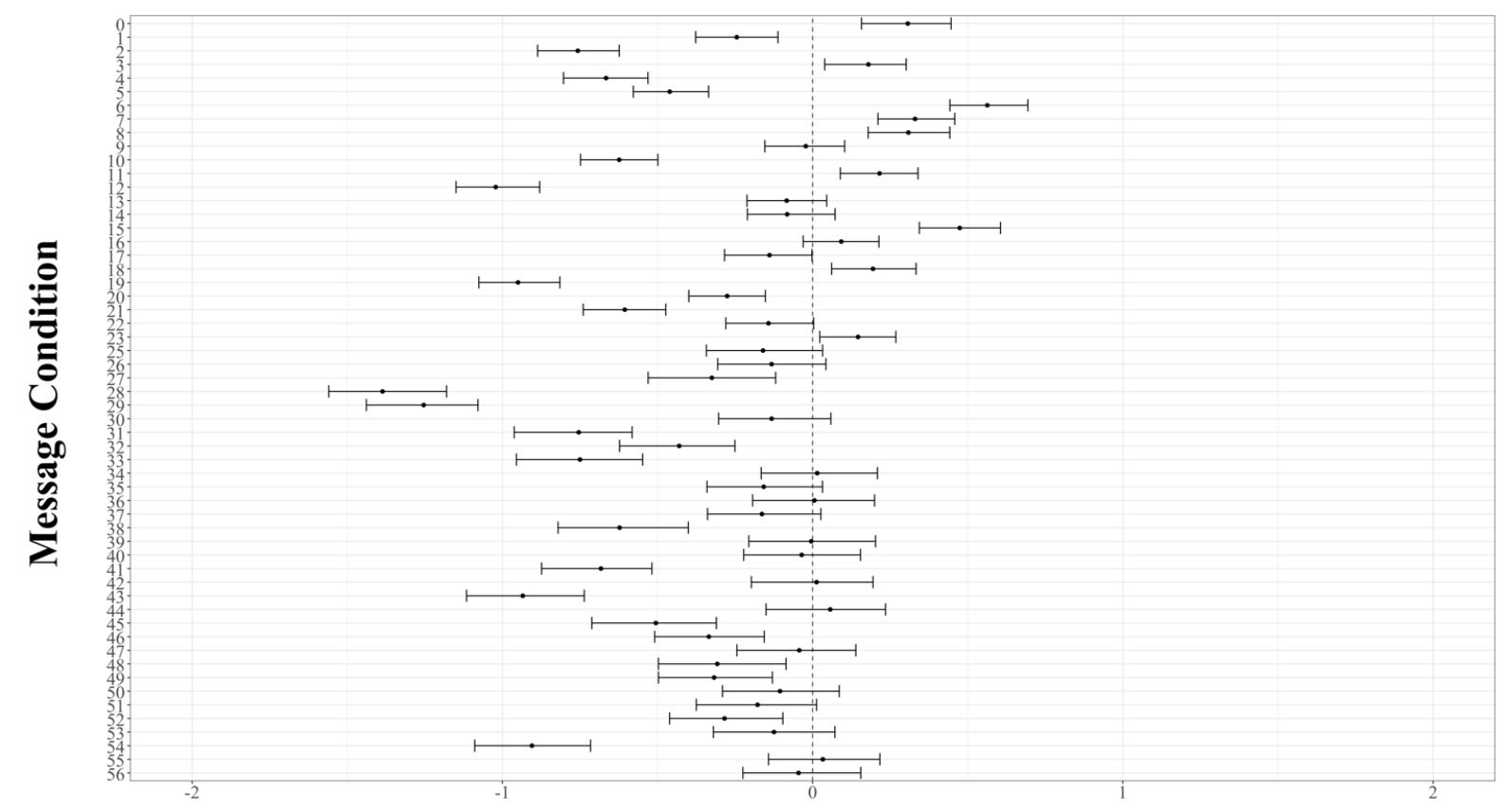

\section{Effect (Regression Coef)}

Figure 1: Effect Size of Messages Relative to Control in Merged Analysis of Studies 1 and 2. (95\% confidence intervals). See Supplementary Materials for message number key.

Table 1: Messages that Outperformed Control in Merged Analysis of Studies 1 and 2

\begin{tabular}{|c|c|c|c|c|}
\hline $\begin{array}{l}\text { Message } \\
\text { Number }\end{array}$ & Text & $\begin{array}{l}\text { Effect size, } \\
\text { Pre-test } 1\end{array}$ & $\begin{array}{l}\text { Effect size, } \\
\text { Pre-test } 2\end{array}$ & $\begin{array}{l}\text { Effect } \\
\text { size, } \\
\text { Merged } \\
\text { Analysis }\end{array}$ \\
\hline 6 & $\begin{array}{l}\text { Doctors, nurses, and other health care workers are } \\
\text { working around the clock, risking their lives to care for } \\
\text { patients with the coronavirus. Working long hours in } \\
\text { highly infectious environments, many of them are falling } \\
\text { ill. As our health care workers put their lives on the line, } \\
\text { we can do our part simply by staying home and limiting } \\
\text { physical contact with others }\end{array}$ & $\begin{array}{l}0.60 * * * \\
(0.11)\end{array}$ & $\begin{array}{l}0.39 * * \\
(0.094)\end{array}$ & $\begin{array}{l}0.54 * * * \\
(0.067)\end{array}$ \\
\hline 15 & $\begin{array}{l}\text { Public health officials tell us that we must slow the } \\
\text { spread of the coronavirus so numbers of sick people } \\
\text { don't overwhelm our doctors, nurses, and hospitals. If } \\
\text { we don't slow the spread, cases will increase rapidly, } \\
\text { suddenly spiking beyond what the health care system } \\
\text { can handle. We all can do our part to slow the spread by } \\
\text { staying inside and avoiding contact with others when we } \\
\text { must go out. If we take action to slow the spread now, } \\
\text { we will save lives. }\end{array}$ & $\begin{array}{l}0.59 * * * \\
(0.11)\end{array}$ & $\begin{array}{l}0.21 \\
(0.094)\end{array}$ & $\begin{array}{l}0.46 * * * \\
(0.067)\end{array}$ \\
\hline 7 & $\begin{array}{l}\text { A few weeks ago, Fiona was a healthy } 26 \text {-year-old with } \\
\text { no medical complications. Then she suddenly came } \\
\text { down with a bad cough and a feeling like she could not } \\
\text { breathe. She tested positive for COVID-19, and is now } \\
\text { hospitalized, receiving oxygen from a ventilator, and } \\
\text { fighting for her life. This could be any of us. Please stay } \\
\text { home and protect yourself against this virus! }\end{array}$ & $\begin{array}{l}0.35 * \\
(0.11)\end{array}$ & $\begin{array}{l}0.19 \\
(0.095)\end{array}$ & $\begin{array}{l}0.32 * * * \\
(0.067)\end{array}$ \\
\hline
\end{tabular}




\begin{tabular}{|l|l|l|l|l|}
\hline 0 & $\begin{array}{l}\text { The sick, elderly, and immuno-compromised need our } \\
\text { help. We all have a choice: If we go out, we risk the } \\
\text { lives of others. But by staying home we can protect those } \\
\text { most likely to be harmed. Stay home to protect those } \\
\text { who are vulnerable! }\end{array}$ & $\begin{array}{l}0.34^{*} \\
(0.11)\end{array}$ & $\begin{array}{l}0.17 \\
(0.095)\end{array}$ & $\begin{array}{l}0.29 * * * \\
(0.066)\end{array}$ \\
\hline 8 & $\begin{array}{l}\text { On average, each person passes on the coronavirus to 2 } \\
\text { to 3 people, who then pass it on to more people, and so } \\
\text { on. If you break a chain of transmission, you can single- } \\
\text { handedly save lives and prevent the suffering of } \\
\text { potentially dozens of people. Stay home as much as you } \\
\text { can, and break the transmission chain! }\end{array}$ & $\begin{array}{l}0.48^{* * *} \\
(0.11)\end{array}$ & $\begin{array}{l}-0.02 \\
(0.092)\end{array}$ & $\begin{array}{l}0.29 * * * \\
(0.067)\end{array}$ \\
\hline
\end{tabular}

Beta values are non-standardized.

All reported p-values are adjusted using the Holm correction for multiple hypothesis testing. $* \mathrm{p}<.05 ; * * \mathrm{p}<.01 ; * * * \mathrm{p}<.001$

\section{Study 1}

In this study, we test whether the four messages that were rated as most persuasive in the previous studies cause people who are not fully compliant to change their behaviours.

\section{Method}

\section{Design and Participants}

This was a pre-registered, 6-condition, between-subjects study, with 4 treatment messages compared to a null control (no message) and an "active control" message that has a reminder of the virus and recommended behaviours. The active control message is the same as the control message in the pre-tests. We use both null control and an active control message in order to distinguish the effect of a specific message from the effect of reminding people of recommended behaviours. The four treatment messages were the messages that were significantly more persuasive than the control in the merged analysis of both pre-tests, and more persuasive than the control in the second pre-test, which focused on low compliance people (Messages \#6, \#7,\#15, and \#0). We made small edits to the messages to bring them up to date with current public health guidelines. 
We used G*Power to calculate that 250 participants per condition would allow us to detect an effect size of Cohen's $d=0.3($ Power $=80 \%$, alpha $=0.05)$. Therefore, we targeted a sample size of 1500 .

The study was fielded between May 22-23, 2020. Participants were recruited from Lucid. 5,180 participants began the survey. After excluding participants who failed a simple attention check as, 4,149 remained (See Supplementary Materials for attention check item).

Only low and mid-level compliers with public health guidelines qualified to complete the survey. That is, people who reported engaging in at least two of the six behaviours listed in Study 2. We made the filter less restrictive because there were not large differences between messages that were convincing to low-compliers and the full population. 1,767 participants passed this filter.

Following pre-registered exclusion criteria, we excluded participants who failed a simple attention check at the end of the survey. There was no differential attrition based on this exclusion. This left 1,627 participants. (48\% male, median age $=47,42 \%$ conservative, $30 \%$ moderate, and $28 \%$ liberal). For the analyses that did not include the null control message, we also excluded participants who spent less than two seconds reading the message to account for inattentive participants, again following our pre-registered exclusion criteria. 1,072 participants met the inclusion criterion across the five conditions that were not the null control.

\section{Procedure}

Change in intended behaviors. After reading the message, participants indicated how often, in the next week, they intended to engage in the same six behaviours that they were asked about earlier (staying at home, wearing a mask, etc.). The items were on the same scale as the filter questions but were about intended future behaviours instead of past behaviours (see 
Supplementary Materials). We took the difference between participants' intended behaviours and past behaviours on each of the six items and normalized each one. The main dependent variable was the average of these six normalized difference scores. We chose this variable because we wanted to measure whether the message caused people to take more precautionary behaviours in the future than they did in the past.

\section{Results}

We ran two multiple linear regressions with the average normalized difference score across the six behaviours as the outcome variable. In the first model, the null control was the reference group. The additional covariates included in the model were 1) normalized measures of age, income, and 2) dummy variables for education level and race/ethnicity. Compared to the null control, three messages and the active control message caused an increase in intentions to comply with public health guidelines, and the one message that was not significant showed trending effects (Table 2).

Next, we conducted our pre-registered analysis. This was the same model, but excluding the null control and instead using the active control as the reference group. None of the four messages caused a significantly greater increase in preventative behaviours intentions than the active control (Table 2).

Table 2: Regression from Study 1, Aggregate Difference Score as Outcome

\begin{tabular}{|l|c|c|}
\hline Variable & $\begin{array}{c}\text { Model 1 } \\
\text { Null control as reference } \\
\text { group }\end{array}$ & $\begin{array}{c}\text { Model 2 } \\
\text { Active control as reference } \\
\text { group }\end{array}$ \\
\hline Active control & $0.106^{*}$ & -- \\
& $(.046)$ & 0.0098 \\
\hline Message 6 & 0.081. & $(.052)$ \\
\hline Message 7 & $(.046)$ & 0.080 \\
& $0.165^{* * *}$ & $(.050)$ \\
\hline Message 15 & $(.045)$ & 0.0695 \\
& $0.159^{* * *}$ & $(.053)$ \\
\hline
\end{tabular}




\begin{tabular}{|c|c|c|}
\hline Message 0 & $\begin{array}{c}0.159 * * * \\
(.047)\end{array}$ & $\begin{array}{l}0.0337 \\
(.053)\end{array}$ \\
\hline Normalized Age & $\begin{array}{c}-0.0159 \\
(.014)\end{array}$ & $\begin{array}{l}-0.024 \\
(.017)\end{array}$ \\
\hline Male & $\begin{array}{c}-0.142 * * * \\
(.027)\end{array}$ & $\begin{array}{c}-.125 * * * \\
(.033)\end{array}$ \\
\hline Education: HS or less & $\begin{array}{c}0.0783 * \\
(0.038)\end{array}$ & $\begin{array}{l}0.091 . \\
(.048)\end{array}$ \\
\hline Education: Some college & $\begin{array}{l}0.033 \\
(.034)\end{array}$ & $\begin{array}{l}0.028 \\
(.042)\end{array}$ \\
\hline Education: Postgraduate & $\begin{array}{l}-.004 \\
(.045) \\
\end{array}$ & $\begin{array}{l}-0.025 \\
(.055) \\
\end{array}$ \\
\hline Race: Asian & $\begin{array}{l}0.077 \\
(.060) \\
\end{array}$ & $\begin{array}{l}0.079 \\
(.076) \\
\end{array}$ \\
\hline Race: Black & $\begin{array}{c}0.212^{* * *} \\
(.047)\end{array}$ & $\begin{array}{c}0.292 * * * \\
(.063)\end{array}$ \\
\hline Race: Hispanic & $\begin{array}{c}0.125 * * \\
(.058)\end{array}$ & $\begin{array}{c}0.159^{*} \\
(.080)\end{array}$ \\
\hline Race: Other & $\begin{array}{l}0.142 \\
(.09)\end{array}$ & $\begin{array}{l}0.152 \\
(.10)\end{array}$ \\
\hline Income & $\begin{array}{c}-4.0 \mathrm{e}-08 \\
(2.8 \mathrm{e}-07)\end{array}$ & $\begin{array}{c}9.2 \mathrm{e}-08 \\
(3.52 \mathrm{e}-07)\end{array}$ \\
\hline R-squared & 0.042 & 0.041 \\
\hline Number of observations & 1627 & 1072 \\
\hline
\end{tabular}

$. \mathrm{p}<.1 ; * \mathrm{p}<.05 ; * * \mathrm{p}<.01 ; * * * \mathrm{p}<.001$

Standard errors are shown in parentheses.

\section{Discussion}

Participants who read any message advocating for COVID-19 preventive behaviours reported significantly greater increases in intentions to engage in preventive behaviours than those in the null control condition. This suggests that seeing a reminder of the pandemic and recommended behaviours has an effect.

However, no messages were significantly more persuasive than the active control message. This suggests that the persuasive strategies had minimal effects.

One possible reason for null results is that we conducted the study at a time when many states were relaxing shelter-in-place orders (25). The messages may have seemed contradictory to "reopening" messaging prevalent at the time. In addition, Message \#6 may not have seemed 
accurate -- several participants expressed suspicion regarding whether healthcare systems were heavily impacted at that time.

\section{Study 2}

We tested the persuasive messages again in Study 2. One concern from Study 1 was that the behaviours the message called for may have been outdated. Stay-at-home orders were being lifted, and most public health officials were advocating for new guidelines, such as wearing face masks. In this study, we kept the same messaging, but modified the recommended behaviours.

\section{Method}

\section{Design and Participants}

Study 2 was a pre-registered, 5-condition study, comparing four treatment messages to an active control message. We adapted messages \#6, \#7, \#15, and \#0 to call for three new behaviours instead of asking people to stay home: physically distancing from others, wearing a mask, and washing hands after returning home (see Supplementary Materials for message texts).

We fielded the study on Mechanical Turk from June 29 - July 1, 2020. At this point, the number of new COVID-19 cases per day had increased beyond the prior peak in April. Using $G^{*}$ Power, we found that a sample of at least 1,530 would allow us to detect a small effect size of Cohen's $d=0.25($ Power $=80 \%$, alpha $=0.05)$.

We excluded participants who failed the same attention checks as in Study 1 - the two simple attention checks as well as those who spent less than 2 seconds reading the message. We also excluded participants who were highly compliant with the three behaviours in the message. Participants reported how often they engaged in the three behaviours when they last left their place of residence, on a scale from 0 (Never) to 100 (Extremely often). We allowed participants to enter the study whose average on these three items was less than or equal to 93 out of 100 . We 
chose this criterion because based on pilot data suggesting $50-60 \%$ of respondents would qualify. We slackened these criteria for two reasons. First, we did not see large differences in which messages were persuasive to the full sample in the first pretest and the low-compilers in the second pretest. Second, it would be beneficial to have 100\% compliance on these behaviours, since participants can comply with them under almost all circumstances. Anyone who is not highly compliant has room for improvement.

3,058 unique respondents began the survey. 1,666 passed the first simple attention check and the filter for low-compliers. Of those, 1,531 passed the end attention check, and 1,421 participants spent at least two seconds on the page with the messages (58\% male, median age $=34,43 \%$ conservative, $19 \%$ moderate, $37 \%$ liberal).

\section{Procedure}

The procedure was similar to that of Study 1, but with new items before the messages to measure respondents' skepticism toward COVID-19.

Skepticism of COVID. Before reading the messages, participants answered two items on scales ranging from 0 (Strongly disagree) to 100 (Strongly agree) measuring how skeptical people were about the severity of COVID-19 (e.g. "Most people are overreacting to COVID19.") $(\alpha=0.93)$.

Difference Score. After reading the messages, participants indicated how often they intended to engage in the three behaviours listed in the message the next time they left their place of residence. We calculated the difference between the intended actions and reported past actions for each activity, and the main dependent variable was the average of these three differences $($ Mean $=5.0$, Median $=7.0)$.

\section{Results}


Consistent with our pre-registration, we ran the same regression model as in Study 3 to identify which messages caused a greater increase in intentions to engage in preventive behaviours. The most persuasive message was Message \#6 $(\beta=2.47, \mathrm{p}=.02)$, which was significantly more persuasive than the active control. No other messages were more persuasive than the active control (Table 3).

Most of the effect of Message \#6 on intended compliance was driven by individuals low in skepticism about COVID-19. Among participants with skepticism rating below 50, those who viewed Message \#6 $(\beta=3.49, \mathrm{p}=.03)$ and Message $\# 15(\beta=3.08, \mathrm{p}=0.05)$ reported a greater increase in intentions to comply. There was no significant effect of any message among participants with skepticism scores greater than 50 .

\section{Discussion}

Message \#6, which was most persuasive in the pre-tests, was significantly more persuasive than the control in Study 2. There are several reasons why these results may have differed from Study 1. First, the messages called for more relevant behaviours, whereas Study 1 messages may have contradicted other information respondents were receiving. Second, cases were rising when Study 2 was fielded (26), so it may have seemed more realistic that hospitals could be overwhelmed.

\section{Study 3}

Given the different effects in Studies 1 and 2, we conducted a replication of Study 2, featuring only Message \#6 and the active control.

\section{Method}

\section{Design and Participants}


This pre-registered study used a two-condition, between-subjects design. Otherwise, the procedure was the same as Study 2. We expected a small effect size, so using G*Power, we found that 620 participants would allow us to detect an effect size of Cohen's $d=0.2(80 \%$ power, alpha $=0.05)$.

The study was fielded on Mechanical Turk from July 14-15, 2020, when the number of new COVID-19 cases reported per day was increasing rapidly (25). Of the 1,212 participants who began the survey, 648 passed the behaviour filter and simple attention item, 597 responded correctly to the end attention check, and 568 spent at least 2 second reading the message (52\% male, median age $=34,46 \%$ conservative, $36 \%$ liberal, $18 \%$ moderate).

\section{Results}

Using the same multiple linear regression as Study 2, Message \#6 was not significantly more persuasive than the control (Table 3). Though we conducted the study under nearly identical conditions approximately two weeks later, we did not find a significant effect of Message \#6 on increase in intentions to comply.

Table 3: Regression from Study 2 and 3, Difference Score as Outcome

\begin{tabular}{|l|l|l|}
\hline Variable & Study 2 & Study 3 \\
\hline Message 6 & $2.47^{*}$ & -0.765 \\
& $(1.08)$ & $(0.89)$ \\
\hline Message 7 & 0.52 & -- \\
& $(1.07)$ & \\
\hline Message 15 & 1.76 & -- \\
& $(1.08)$ & \\
\hline Message 0 & 1.11 & -- \\
& $(1.08)$ & \\
\hline Normalized Age & -0.21 & 2.82 \\
& $(0.328)$ & $(0.5)$ \\
\hline Gender: Male & $-1.44^{*}$ & $-2.64^{* *}$ \\
& $(0.69)$ & $(0.91)$ \\
\hline Education: HS or less & $2.7^{*}$ & 1.45 \\
& $(1.25)$ & $(1.64)$ \\
\hline Education: Some college & $2.35^{* *}$ & 1.00 \\
& $(0.85)$ & $(1.15)$ \\
\hline Education: Post-graduate & 0.66 & 1.27 \\
& $(0.94)$ & $(1.27)$ \\
\hline
\end{tabular}




\begin{tabular}{|c|c|c|}
\hline Race: Asian & $\begin{array}{l}2.56^{*} \\
(1.25)\end{array}$ & $\begin{array}{l}1.41 \\
(1.57)\end{array}$ \\
\hline Race: Black & $\begin{array}{l}0.584 \\
(1.12)\end{array}$ & $\begin{array}{l}1.12 \\
(1.41)\end{array}$ \\
\hline Race: Hispanic & $\begin{array}{l}0.62 \\
(1.77)\end{array}$ & $\begin{array}{l}3.96 \\
(1.73)\end{array}$ \\
\hline Race: Other & $\begin{array}{l}-1.92 \\
(3.07)\end{array}$ & $\begin{array}{l}4.84 \\
(5.35)\end{array}$ \\
\hline Income & $\begin{array}{l}-6.24 \mathrm{e}-06 \\
(7.99 \mathrm{e}-06)\end{array}$ & $\begin{array}{l}2.5 e-6 \\
(9.4 e-6)\end{array}$ \\
\hline R-squared & 0.01 & 0.003 \\
\hline Number of observations & 1427 & 568 \\
\hline
\end{tabular}

$* \mathrm{p}<.05 ; * * \mathrm{p}<.01, * * * \mathrm{p}<.001$

Standard errors are shown in parentheses.

\section{Discussion}

The null effect may be because the earlier evidence for Message \#6 were false positives. Alternatively, other changes in the world - e.g., crystallization of Americans' views of the pandemic - may have led this message to no longer increase intentions to engage in preventive behaviours.

\section{Data Availability}

Preregistration, data, code, and materials for this and the following studies can be found here: https://osf.io/eqr4w/?view_only=4865baa4a60846339c779ec2565f463b.

\section{General Discussion}

Across two within-subjects pre-tests where participants rated the persuasiveness of many short messages, several messages were significantly more persuasive than a control message.

We tested four messages in a between-subjects study, and all four led to an increase in intentions to comply with public health guidelines, compared to seeing no message. This suggests that seeing several different types of reminders of the virus and behaviours can increase intentions to engage in prevention behaviours. However, across three studies, none of the four messages consistently led to an increase in intentions to comply with public health guidelines 
compared to a standard public health message. We find that changes in the persuasive approach from short messages do not significantly affect participants' intentions to increase compliance with public health guidelines. Message \#6, which emphasized civic responsibility to reciprocate sacrifices made by healthcare workers, was rated most persuasive in the pre-tests, out of 24 and 56 messages, respectively. It also showed a significant effect in Study 2. However, it did not show an effect in a pre-registered replication.

We can only speculate why no messages were consistently more persuasive than a standard public health message. Coronavirus was in the news constantly and impacted almost every American. Small differences between short messages may not be persuasive in such a saturated information environment. It could be there was a "persuadable window" early on when short messages could change behaviours, but the messages were no longer effective later on due to crystallization of views, which is similar to other COVID-19 messaging work (16). Research on the effectiveness of political ads, another salient issue, has also found that political ads for Presidential candidates have minimal effects (27). In addition, we found that many messages in the pretests were rated as significantly less persuasive than a control message. Messages evoking national identity, collective action, patriotism, analogies to war, religion, and purity were not perceived as highly persuasive. Likewise, messages highlighting vivid costs of not following guidelines (e.g., killing others, citations by law enforcement) were generally perceived to be less persuasive than the control. It may be that these messages read as direct persuasive efforts for certain subgroups, and prior work on direct vs. peripheral appeals suggest that some people often resist direct appeals (28).

\section{Limitations}


These experiments were conservative tests of messaging because their brevity may have lead to minimal impact on participants. Participants read a very short message, without a clear source, one time. The messages might be more persuasive if they were longer, delivered multiple times, or were conveyed via a more compelling medium, such as video. Similarly, one-on-one conversations (29) or receiving the message from a credible source (30) might have been more persuasive than decontextualized messages. It is possible that some of the persuasive strategies from the messages are effective, but only if delivered differently.

Another limitation is that we relied on self-reported past and intended behaviours. Despite our care to include active controls, our dependent variable may still be susceptible to social desirability bias. In addition, the pandemic affected different regions of the country at different points in time, and health guidelines varied substantially by region. Some messages may have been effective in certain regions at certain times, but our studies were insufficiently powered to analyze regional differences.

\section{Conclusion}

Across two pre-tests and three experiments conducted throughout five months of the coronavirus pandemic, results on the persuasiveness of different messaging strategies were inconsistent. We found that seeing a message with any reminder of the virus and prevention behaviours outperformed seeing no message at all. However, across three studies, none of the top-rated messages consistently outperformed a standard public health message. We generally

found that the highest performing message highlighted reciprocity towards healthcare workers, but this effect did not replicate in the final study. Messages that are more frequent, more vivid, or from more credible sources may be more effective at increasing intentions to engage in 
prevention behaviours. These findings suggest that small changes in messaging are largely incapable of affecting intended compliance during advanced stages of the pandemic.

\section{Author Contributions Statement}

All authors contributed to the study concept and study design. Data collection was performed by J.M. and S.P. S.P. performed data analysis with assistance from J.V. S.P drafted the manuscript, with critical revisions from all authors.

\section{Competing Interests Statement}

The authors declare no competing interests.

\section{References}

1. Eikenberry, S. E., et al. To mask or not to mask: Modeling the potential for face mask use by the general public to curtail the COVID-19 pandemic. Infectious Disease Modelling, 5, 293308 (2020).

2. Snyder, L.B. et al. A Meta-Analysis of the Effect of Mediated Health Communication Campaigns on Behavior Change in the United States. Journal of Health Communication. 4, 71-96 (2004).

3. Stead, M. et al. Mass media to communicate public health messages in six health topic areas: a systematic review and other reviews of the evidence. Public Health Research 7 (2019)

4. Hall, A.K., Cole-Lewis, H. \& Bernhardt, J.M. Mobile Text Messaging for Health: A Systematic Review of Reviews. Annual Review of Public Health. 36, 393-415 (2015).

5. Keller, P. A., \& Lehmann, D. R. Designing effective health communications: A metaanalysis. Journal of Public Policy \& Marketing, 27(2), 117-130 (2008).

6. Shen, F., Sheer, V. C., \& Li, R. Impact of narratives on persuasion in health communication: A meta-analysis. Journal of Advertising, 44, 105-113 (2015).

7. Kellens, W., Terpstra, T., \& De Maeyer, P. Perception and communication of flood risks: a systematic review of empirical research. Risk Analysis: An International Journal, 33(1), 2449 (2013).

8. Cialdini, R. B. Influence (Vol. 3). Port Harcourt: A. Michel. (1987).

9. Brenan, M. Americans' trust in mass media edges down to $41 \%$. Gallup. https://news.gallup.com/poll/267047/americans-trust-mass-media-edges-down.aspx (2019).

10. Pew Research Center. Public Trust in Government: 1958-2019. Pew Research Center - U.S. Politics \& Policy. https://www.pewresearch.org/politics/2019/04/11/public-trust-ingovernment-1958-2019/ (2019). 
11. Newport, F. The partisan gap in views of the coronavirus. Gallup. https://news.gallup.com/opinion/polling-matters/311087/partisan-gap-viewscoronavirus.aspx (2020).

12. Schaeffer, K. Nearly three-in-ten Americans believe COVID-19 was made in a lab. Pew Research Center. https:/www.pewresearch.org/fact-tank/2020/04/08/nearly-three-in-tenamericans-believe-covid-19-was-made-in-a-lab/ (2020)/

13. Morrison, F. P., Kukafka, R., \& Johnson, S. B. Analyzing the structure and content of public health messages. American Medical Informatics Association Annual Symposium Proceedings 2005, 540 American Medical Informatics Association. (2005).

14. Noar, S. M. A 10-year retrospective of research in health mass media campaigns: where do we go from here?. Journal of health communication, 11(1), 21-42. (2006)

15. Grant, A. M., \& Hofmann, D. A. It's not all about me: Motivating hand hygiene among health care professionals by focusing on patients. Psychological Science, 22(12), 1494-1499. (2011).

16. Jordan, J., Yoeli, E., \& Rand, D. G. Don't get it or don't spread it? Comparing self-interested versus prosocial motivations for COVID-19 prevention behaviors [Preprint]. PsyArXiv. (2020).

17. Hendrix et al. Vaccine Message Framing and Parents' Intent to Immunize Their Infants for MMR. Pediatrics. 134(3) e675-e683 (2014).

18. Small, D. A., Loewenstein, G., \& Slovic, P. Sympathy and callousness: The impact of deliberative thought on donations to identifiable and statistical victims. Organizational Behavior and Human Decision Processes, 102(2), 143-153 (2007).

19. Schultz, P. W., Nolan, J. M., Cialdini, R. B., Goldstein, N. J., \& Griskevicius, V. The constructive, destructive, and reconstructive power of social norms. Psychological Science, 18(5), 429-434. (2007).

20. Cialdini, R. B., Green, B. L., \& Rusch, A. J. When tactical pronouncements of change become real change: The case of reciprocal persuasion. Journal of Personality and Social Psychology, 63(1), 30. (1992).

21. Feinberg, M., \& Willer, R. The moral roots of environmental attitudes: Psychological Science. 24.1 56-62 (2013).

22. Feinberg, M., \& Willer, R. Moral reframing: A technique for effective and persuasive communication across political divides. Social and Personality Psychology Compass, 13(12), e12501. (2019).

23. Luttrell, A., \& Petty, R. E. Evaluations of Self-Focused versus Other-Focused Arguments for Social Distancing: An Extension of Moral Matching Effects. Social Psychological and Personality Science (2020).

24. Everett, J. A. C., Colombatto, C., Chituc, V., Brady, W. J., \& Crockett, M. The effectiveness of moral messages on public health behavioral intentions during the COVID-19 pandemic [Preprint]. PsyArXiv (2020). 
25. Iati, M, et al. "All 50 U.S. States Have Taken Steps toward Reopening in Time for Memorial Day Weekend.” Washington Post, https://www.washingtonpost.com/nation/2020/05/19/coronavirus-update-us/. (2020).

26. CDC. Coronavirus Disease 2019 (COVID-19) in the US. Centers for Disease Control and Prevention. https://www.cdc.gov/coronavirus/2019-ncov/cases-updates/cases-in-us.html (2020).

27. Coppock, A., Hill, S. J., \& Vavreck, L.The small effects of political advertising are small regardless of context, message, sender, or receiver: Evidence from 59 real-time randomized experiments. Science Advances, 6(36) (2020).

28. Cacioppo, J. T., Petty, R. E., Kao, C. F., \& Rodriguez, R. Central and peripheral routes to persuasion: An individual difference perspective. Journal of personality and social psychology, 51(5), 1032 (1986).

29. Broockman, D., \& Kalla, J. (2016). Durably reducing transphobia: A field experiment on door-to-door canvassing. Science, 352(6282), 220-224. (2016).

30. Turner, J. C., Hogg, M. A., Oakes, P. J., Reicher, S. D., \& Wetherell, M. S. Rediscovering the social group: A self-categorization theory. Basil Blackwell. (1987) 